\title{
Fotossíntese e potencial hídrico foliar de plantas jovens de andiroba submetidas à deficiência hídrica e à reidratação
}

\author{
José Francisco de Carvalho Gonçalves ${ }^{(1)}$, Carlos Eduardo Moura da Silva ${ }^{(1)}$ e Diogo Gato Guimarães ${ }^{(1)}$ \\ (1)Instituto Nacional de Pesquisas da Amazônia, Laboratório de Fisiologia e Bioquímica Vegetal, Caixa Postal 476, CEP 69011-970 Manaus, AM. \\ E-mail: jfc@inpa.gov.br, cems@inpa.gov.br, diogo_gato44@hotmail.com
}

\begin{abstract}
Resumo - O objetivo deste trabalho foi investigar o desempenho fotossintético de plantas jovens de andiroba (Carapa guianensis), submetidas à deficiência hídrica e à reidratação. As plantas foram irrigadas diariamente, em casa de vegetação, durante 15 dias. Após aclimatação, as plantas foram separadas em dois tratamentos: plantas irrigadas e não irrigadas. Quando a resposta fotossintética das plantas não irrigadas alcançou valores próximos a zero, as plantas foram reidratadas. A cada sete dias, durante 21 dias, foram realizadas as determinações das trocas gasosas e da fluorescência da clorofila $a$. O potencial hídrico foliar foi determinado no início e no fim do experimento. As taxas de fotossíntese líquida, condutância estomática e transpiração se reduziram em 88,89 e $89 \%$, respectivamente, após 21 dias de supressão da irrigação. Quanto às variáveis da fluorescência da clorofila $a$, observaram-se reduções de 27 a $58 \%$. O potencial hídrico foliar das plantas foi reduzido em mais de quatro vezes, após 21 dias de deficiência hídrica. De quatro a oito dias após a reidratação, as plantas recuperaram as características fotossintéticas e o potencial hídrico foliar, o que indica que plantas jovens de andiroba apresentaram alta plasticidade fisiológica em relação ao estresse hídrico.
\end{abstract}

Termos para indexação: Carapa guianensis, deficit hídrico, espécie arbórea, fluorescência da clorofila $a$, trocas gasosas.

\section{Photosynthesis and water potential of andiroba seedlings submitted to water stress and rewetting}

\begin{abstract}
The objective of this work was to investigate the photosynthetic performance of young andiroba plants (Carapa guianensis) submitted to water stress and rewetting. The plants were irrigated daily in greenhouse conditions for 15 days. After acclimatization, the plants were separated randomly in two treatments, irrigated and non-irrigated plants. When the photosynthesis of the non-irrigated plants reached values close to zero, the plants were rewetted. Measurements of gas exchanges and chlorophyll $a$ fluorescence were recorded on seven-day intervals, during 21 days. Plant water potentials were determined at the beginning and at the end of the experiment. Net photosynthesis, stomatal conductance, and transpiration rates were reduced by 88,89 , and $89 \%$, respectively, after 21 days of water stress. In relation to chlorophyll $a$, fluorescence variables were reduced 27 to $58 \%$. The plant water potential was reduced more than four times by 21 days of stress. With rewetting, seedlings recovered their photosynthetic characteristics in four to eight days as well as their water potential, indicating that young andiroba plants exhibited high physiological plasticity to water stress.
\end{abstract}

Index terms: Carapa guianensis, water deficit, tree species, chlorophyll $a$ fluorescence, gas exchange.

\section{Introdução}

Aspectos relacionados à fisiologia das árvores tropicais têm recebido grande atenção, uma vez que modificações no cenário florestal (desmatamentos, queimadas, degradação de solos) refletem diretamente no funcionamento das plantas e contribuem para criar incertezas no planejamento de programas de manejo florestal sustentável ou de produção florestal (plantios de oleaginosas arbóreas para indústria de fármacos, cosméticos ou de biocombustível) (Gebrekirstos et al., 2006; Reinhardt et al., 2007).

Alterações nos fatores abióticos afetam sobremaneira a atividade fisiológica das plantas em suas diferentes fases de crescimento. Fatores como alta irradiância e baixa disponibilidade hídrica ou nutricional tornam mais difícil o sucesso no estabelecimento das plantas na fase juvenil 
(Gonçalves et al., 2005; Liberato et al., 2006; Santos Junior et al., 2006).

Em relação à disponibilidade hídrica, na floresta Amazônica o regime pluvial é marcado pela ocorrência de altas precipitações, que variam de 2.300 a $3.500 \mathrm{~mm}$ porano. No entanto, a parte central da RegiãoAmazônica está sob o domínio da Célula de Hadley, que impõe um período bem característico de seca (Fisch et al., 1998). A disponibilidade hídrica é um fator determinante para a abundância e distribuição das plantas nos diversos ecossistemas (Farquhar \& Sharkey, 1982), e tem sido considerada um fator seletivo em ambientes onde é baixa (Chaves et al., 2002).

Pesquisas sobre deficit hídrico, em diferentes espécies vegetais, têm sido realizadas por décadas, a fim de se entender os mecanismos de tolerância (O’Leary, 1970; Turner, 1981; Casper et al., 1993; Calbo \& Moraes, 2000; Silva et al., 2003; Liberato et al., 2006; Lechner et al., 2008). Os resultados dessas pesquisas têm sido aplicados em diferentes projetos de manejo de plantios florestais e podem aperfeiçoar as técnicas silviculturais de espécies como a andiroba (Carapa guianensis Aubl.) - árvore tropical da família das Meliaceae, com elevada importância econômica na Amazônia, tanto para a utilização de sua madeira quanto para o uso do óleo extraído das suas sementes, com potencial para uso na indústria de cosméticos e fármacos (Mendonça \& Ferraz, 2007).

O objetivo deste trabalho foi investigar o desempenho fotossintético de plantas jovens de C. guianensis, submetidas à deficiência hídrica induzida e à reidratação.

\section{Material e Métodos}

O experimento foi realizado em casa de vegetação, no Instituto Nacional de Pesquisas da Amazônia, Manaus, AM ( $3^{\circ} 8^{\prime} \mathrm{S}$ e $\left.59^{\circ} 52^{\prime} \mathrm{W}\right)$. Plantas jovens de $C$. guianensis (três meses de idade) oriundas de matrizes da Reserva Florestal Adolpho Ducke, Manaus, que apresentavam uniformidade de tamanho (aproximadamente $35 \mathrm{~cm}$ de altura), foram transplantadas para vasos de plástico (30x25 cm) com capacidade para $8 \mathrm{~kg}$, com mistura de terriço vegetal e argila na proporção de $3: 1$.

Durante 15 dias as plantas foram irrigadas, diariamente, até a capacidade de campo, e aclimatadas à luz $\left(\right.$ máximo $\left.=1.000 \mu \mathrm{mol} \mathrm{m}^{-2} \mathrm{~s}^{-1}\right)$ e à temperatura $\left(30 \pm 8^{\circ} \mathrm{C}\right)$. Após o período de aclimatação, as plantas foram separadas, aleatoriamente, em dois tratamentos: plantas irrigadas e não irrigadas $(\mathrm{n}=10)$. Os indivíduos do tratamento plantas irrigadas receberam irrigação, diariamente, às 8 e $16 \mathrm{~h}$, durante todo o período experimental ( 29 dias). No tratamento plantas não irrigadas, a irrigação foi suspensa até a resposta fotossintética alcançar valores próximos a zero. A reidratação das plantas do tratamento sem irrigação foi realizada até as plantas recuperaram suas características fotossintéticas e o status hídrico inicial. Em ambos tratamentos, foram determinadas as variáveis relacionadas ao potencial hídrico foliar, às trocas gasosas e à fluorescência da clorofila $a$, em intervalos de quatro a sete dias, até o término do experimento.

O potencial hídrico foliar $\left(\Psi_{W}\right)$ foi determinado entre 9 e 11h, utilizando-se uma bomba de pressão tipo Scholander. A determinação consistiu na coleta de amostras de folhas completamente expandidas e com bom estado fitossanitário, as quais foram colocadas na câmara da bomba de pressão, onde, em seguida, foi aplicada pressão até ocorrer a exsudação pelo corte feito no pecíolo da folha, para a leitura da pressão aplicada (Turner, 1981). As leituras foram realizadas após a aclimatação das plantas, quando as respostas fotossintéticas apresentavam valores próximos de zero $(\mathrm{A} \cong 0)$, e no fim do experimento, após a reidratação das plantas.

As determinações das taxas de fotossíntese líquida (A), condutância estomática $\left(\mathrm{g}_{\mathrm{s}}\right)$ e transpiração (E) foram realizadas por meio de um analisador de gás infravermelho (Irga) portátil, de sistema aberto, modelo LICOR 6400, conforme descrito por Santos Junior et al. (2006). As medições foram realizadas entre 8 e $15 \mathrm{~h}$, em folhas completamente expandidas e com bom estado fitossanitário. As respostas das variáveis fotossintéticas à intensidade luminosa foram determinadas para densidade de fluxo de fótons (PPFD) de $1.000 \mu \mathrm{mol} \mathrm{m} \mathrm{m}^{-2} \mathrm{~s}^{-1}$, com a câmara foliar ajustada para trabalhar com concentração de $\mathrm{CO}_{2}$ de $380 \pm 5 \mu \mathrm{mol} \mathrm{mol}{ }^{-1}$, temperatura de $31 \pm 1^{\circ} \mathrm{C}$ e vapor de $\mathrm{H}_{2} \mathrm{O}$ de $21 \pm 1 \mathrm{mmol} \mathrm{mol}^{-1}$. A eficiência do uso da água (EUA) foi calculada por meio da razão A/E.

A determinação da fluorescência da clorofila $a$ foi realizada por meio de um fluorômetro portátil (Plant Efficiency Analyser - MK2 - 9600, Hansatech, Norfolk, UK) entre 8 e 15h, em folhas completamente expandidas e aclimatadas ao escuro por $20 \mathrm{~min}$. Em seguida, as folhas foram expostas a um pulso de luz saturante durante $5 \mathrm{~s}$, numaintensidadede $3.000 \mu \mathrm{molm}^{-2} \mathrm{~s}^{-1}$, 
para se determinar: a eficiência máxima do fotossistema II $\left[\mathrm{TR}_{0} / \mathrm{ABS}=\mathrm{F}_{\mathrm{v}} / \mathrm{F}_{\mathrm{m}}=1-\left(\mathrm{F}_{\mathrm{o}} / \mathrm{F}_{\mathrm{m}}\right)\right]$; a probabilidade de um fóton absorvido mover um elétron após a quinona $\mathrm{A}\left[\mathrm{ET}_{0} / \mathrm{ABS}=\left(1-\mathrm{F}_{\mathrm{o}} / \mathrm{F}_{\mathrm{m}}\right)\left(1-\mathrm{V}_{\mathrm{j}}\right)\right]$; a probabilidade de um elétron capturado ser transportado após a quinona A $\left(\mathrm{ET}_{0} / \mathrm{TR}_{0}=1-\mathrm{V}_{\mathrm{j}}\right)$ e o índice de desempenho $\left\{\mathrm{PI}_{\mathrm{ABS}}=(\mathrm{RC} / \mathrm{ABS})[\varphi \mathrm{Po} /(1-\varphi \mathrm{Po})]\left[\Psi_{0} /\left(1-\Psi_{0}\right)\right]\right\}$. $\mathrm{O} \mathrm{PI}_{\mathrm{ABS}}$ é uma variável da fluorescência da clorofila que fornece informações quantitativas sobre o estado da planta e sua vitalidade, de acordo com o princípio da equação de Nernst (Srivastava et al., 1999). A absorbância (ABS) refere-se à absorção de fótons pela molécula de clorofila no complexo antena. Quando a energia é absorvida pela molécula de clorofila, parte é capturada $\left(\mathrm{TR}_{0}\right)$ pelo centro de reação $(\mathrm{RC})$ do fotossistema II $\left(\mathrm{P}_{680}\right)$, enquanto outra parte é dissipada $\left(\mathrm{DI}_{0}\right)$ na forma de calor ou fluorescência. Da energia capturada, parte é dirigida para a redução de energia química pelo transporte de elétrons $\left(\mathrm{ET}_{0}\right)$ através das quinonas A e B (Force et al., 2003).

$\mathrm{O}$ delineamento experimental utilizado foi $\mathrm{o}$ inteiramente casualizado, com dois tratamentos: plantas irrigadas e não irrigadas, com dez repetições $(n=10)$, considerando cada planta como uma repetição. Os resultados obtidos foram analisados estatisticamente pelo teste de normalidade Lilliefors, seguido pelo teste $t$ de Student (duas amostras relacionadas), a 5\% de probabilidade. O programa utilizado foi o JMP 4.0 (SAS Institute, 2000).

\section{Resultados e Discussão}

O potencial hídrico foliar $\left(\Psi_{\mathrm{w}}\right)$ médio das plantas de C. guianensis, no início do experimento, foi-0,8 MPa. Após 21 dias de suspressão da irrigação o valor decresceu para $-3,4 \mathrm{MPa}$, ao passo que, nas plantas irrigadas o valor observado foi de $-0,7 \mathrm{MPa}$, no mesmo período, o que representa redução do $\Psi_{\mathrm{w}}(\mathrm{p}<0,05)$ de mais de quatro vezes, quando comparado aos das plantas não irrigadas. Costa \& Marenco (2007), ao investigar as trocas gasosas e o potencial hídrico (método psicrométrico) também em plantas de C. guianensis, em árvores de $6 \mathrm{~m}$, em condições de campo, aparentemente sem estresse hídrico, encontraram valores mais elevados de $\Psi_{\mathrm{w}}$, cerca de $-0,4 \mathrm{MPa}$, quando comparado com os valores de plantas irrigadas, no mesmo período diário de leitura (9 às 11h). Vários fatores podem ter concorrido para essa diferença: as condições experimentais, o estádio de crescimento da planta e o método empregado para análise do $\Psi_{\mathrm{w}}$. Por sua vez, Liberato et al. (2006), ao analisar o potencial hídrico e as características fotossintéticas em plantas jovens de Minquartia guianensis Aubl. sob deficiência hídrica, após 31 dias de tratamento, encontraram valores de $\Psi_{\mathrm{w}}$ em torno de -3,2 $\mathrm{MPa}$, quando a taxa de fotossíntese (A) atingiu valores próximos a zero, os quais foram próximos aos encontrados neste trabalho, nas plantas de $C$. guianeneis não irrigadas $(-3,4 \mathrm{MPa})$. No entanto, deve-se ressaltar que nas plantas de $C$. guianensis, além dos valores do $\Psi_{\mathrm{w}}$ observados terem sido menores, a taxa de A próximo de zero ocorreu em menor tempo de supressão da irrigação (21 dias), indicando que C. guianeneis é mais suceptível à deficiência hídrica do que a $M$. guianensis.

Os valores médios da fotossíntese líquida (A) das plantas de C. guianensis irrigadas variaram de 4,1 a $5,3 \mu \mathrm{mol} \mathrm{m} \mathrm{m}^{-2} \mathrm{~s}^{-1}$, durante 21 dias de experimento (Figura 1). Nas plantas não irrigadas, os valores variaram de 3,7 a $0,4 \mu \mathrm{mol} \mathrm{m} \mathrm{m}^{-2} \mathrm{~s}^{-1}$, no mesmo período. Após 14 dias de supressão da irrigação, o valor médio de A foi $0,9 \mu \mathrm{mol} \mathrm{m}^{-2} \mathrm{~s}^{-1}$, e representou redução de $76 \%$,
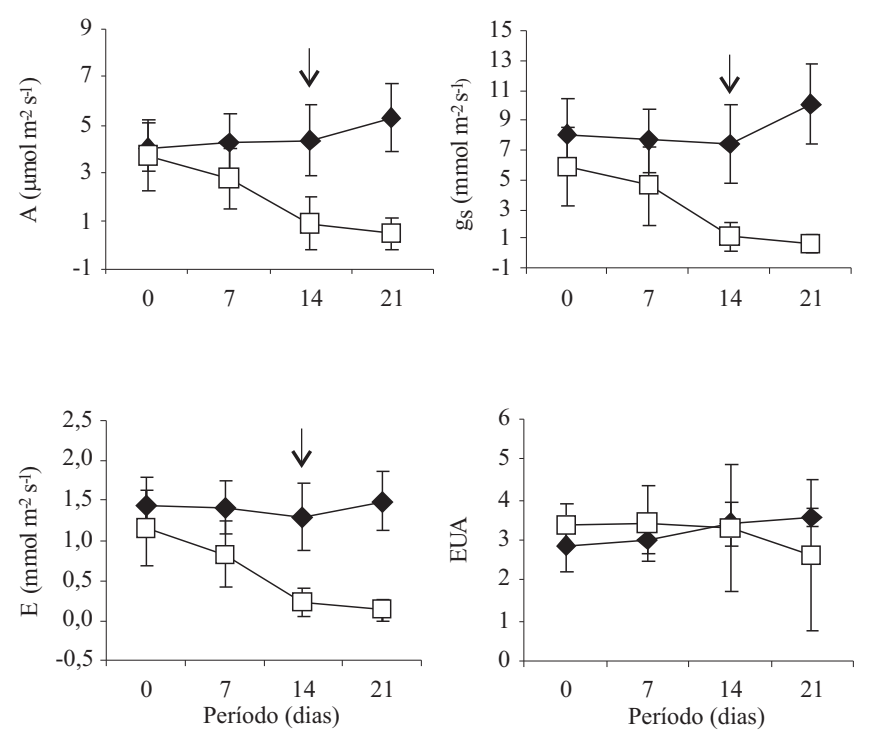

Figura 1. Fotossíntese líquida (A), condutância estomática (gs), transpiração (E) e eficiência no uso da água (EUA) de plantas jovens de Carapa guianensis irrigadas ( $\bullet$ e não irrigadas ( $\square$ ). Os valores são médias e as linhas verticais indicam os desvios-padrão. A seta indica o início da diferença significativa entre os tratamentos pelo teste t, a $5 \%$ de probabilidade $(n=5)$. 
comparado com a leitura inicial $\left(3,7 \mu \mathrm{mol} \mathrm{m} \mathrm{m}^{-2} \mathrm{~s}^{-1}\right)$, e uma diferença de $80 \%$, comparado à resposta das plantas irrigadas $\left(4,4 \mu \mathrm{mol} \mathrm{m} \mathrm{m}^{-2} \mathrm{~s}^{-1}\right)$. Com relação aos valores da condutância estomática $\left(\mathrm{g}_{\mathrm{s}}\right)$, após 14 dias de supressão da irrigação, observou-se diferença significativa entre os tratamentos. Quando os valores de A no tratamento de plantas não irrigadas apresentavam-se próximos de zero, a resposta da $\mathrm{g}_{\mathrm{s}}$ foi de $0,7 \mathrm{mmol} \mathrm{m}^{-2} \mathrm{~s}^{-1}$, representando redução de $89 \%$, quando comparado com a leitura inicial $\left(5,9 \mathrm{mmol} \mathrm{m}^{-2} \mathrm{~s}^{-1}\right)$, e uma diferença de $93 \%$, quando comparado com o tratamento de plantas irrigadas $\left(10,1 \mathrm{mmol} \mathrm{m}^{-2} \mathrm{~s}^{-1}\right)$. Quanto à transpiração (E), observou-se um padrão de respostas semelhantes às respostas da taxa de $\mathrm{g}_{\mathrm{s}}$ durante $\mathrm{o}$ período de supressão da irrigação, porém, com diferença de $91 \%$ no último dia, quando comparado com as plantas do tratamento irrigado. Quanto à eficiência no uso da água (EUA), não se observou diferença significativa entre os tratamentos ao longo do período do experimento.

O fechamento estomático, de fato, representou a causa primária da redução na taxa fotossintética e na taxa de transpiração sob condições de deficiência hídrica, e essa redução deveu-se à diminuição na disponibilidade de $\mathrm{CO}_{2}$ nas câmaras subestomáticas das folhas, causada pelo fechamento dos estômatos. Assim, o decréscimo na disponibilidade hídrica ocasiona queda no potencial da água nas folhas das plantas, levando à perda de turgescência e, consequentemente, à redução da condutância estomática (Flexas \& Medrano, 2002). Dessa forma, a diminuição nas respostas fotossintéticas de C. guianensis, com a supressão da irrigação, pode estar diretamente associada à modificação do comportamento estomático. Evidência dessa natureza, para a mesma espécie, também foi mencionada por Costa \& Marenco (2007). Resultados encontrados neste trabalho com $C$. guianensis $\mathrm{e}$ também observados em plantas M. guianensis, sob condições experimentais semelhantes, evidenciam a variabilidade de respostas apresentada pelas diferentes espécies arbóreas, quando submetidas à supressão da irrigação. No caso da M. guianensis, 25 dias foram suficientes para se observar redução significativa de 25 , 50 e $50 \%$ nas respostas de $\mathrm{A}, \mathrm{E}$ e $\mathrm{g}_{\mathrm{s}}$, respectivamente, comparado ao tratamento com irrigação (Liberato et al., 2006). Em estudo com Bactris gasipaes Kunth, observou-se redução de $40 \%$ na taxa de A no sexto dia de supressão da irrigação, quando o $\Psi_{\mathrm{w}}$ foi igual a $-1,6 \mathrm{MPa}$, além de reduções de 31 e $54 \%$ nas taxas de $\mathrm{E}$ e $\mathrm{g}_{\mathrm{s}}$, no quinto dia de experimento (Oliveira et al., 2002). Em Vitis labrusca L., sete dias foram suficientes para se verificar diferença significativa nas trocas gasosas (A, E e $\mathrm{g}_{\mathrm{s}}$ ) entre os tratamentos de plantas irrigadas e não irrigadas (Souza et al., 2001).

Um aspecto relevante verificado no presente trabalho foi a alta eficiência no uso da água pelas plantas jovens de $C$. guianensis, uma vez que não foi observada diferença significativa entre os tratamentos de plantas irrigadas e não irrigadas. Esses resultados apontam para uma regulação das perdas de água acoplada ao ganho de carbono nas duas situações hídricas (alta e baixa disponibilidade).

Nas plantas jovens de C. guianensis, após 14 dias de supressão da irrigação, foram observadas reduções de $23 \%$ na eficiência máxima do fotossistema II $\left(\mathrm{TR}_{0} / \mathrm{ABS}\right), 39 \%$ na probabilidade de um fóton absorvido mover um elétron após a quinona $\mathrm{A}\left(\mathrm{ET}_{0} / \mathrm{ABS}\right)$, $26 \%$ na probabilidade de um elétron capturado ser transportado após a quinona $\mathrm{A}\left(\mathrm{ET}_{0} / \mathrm{TR}_{0}\right)$ e $56 \%$ no índice de desempenho $\left(\mathrm{PI}_{\mathrm{ABS}}\right)$ (Figura 2). Do ponto de
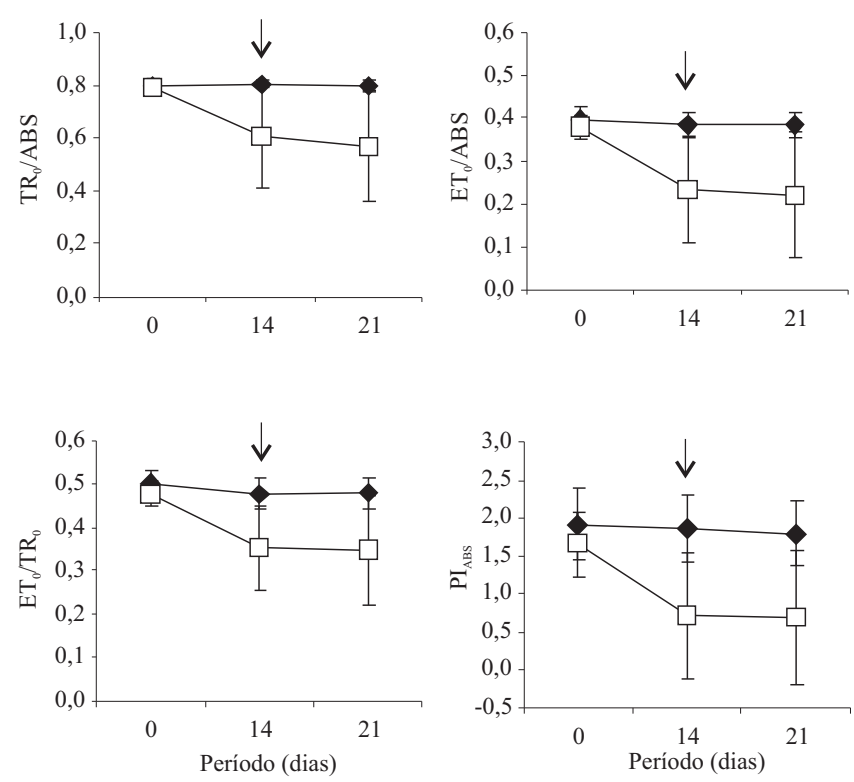

Figura 2. Eficiência máxima do fotossistema II ( $\left.\mathrm{TR}_{0} / \mathrm{ABS}\right)$, probabilidade de um fóton absorvido mover um elétron após a quinona $\mathrm{A}\left(\mathrm{ET}_{0} / \mathrm{ABS}\right)$, probabilidade de um elétron capturado ser transportado após a quinona $\mathrm{A}\left(\mathrm{ET}_{0} / \mathrm{TR}_{0}\right)$ e o índice de desempenho $\left(\mathrm{PI}_{\mathrm{ABS}}\right)$ de plantas jovens de Carapa guianensis irrigadas ( ) e não irrigadas ( $\square)$. Os valores são médias e as linhas verticais indicam os desvios-padrão. A seta indica o início da diferença significativa entre os tratamentos pelo teste $\mathrm{t}$, a $5 \%$ de probabilidade $(\mathrm{n}=5)$. 
vista funcional, os resultados demonstraram queda na regulação da atividade fotoquímica com o progresso da desidratação das plantas de C. guianensis. Contudo, redução de $23 \%$ na $\mathrm{TR}_{0} / \mathrm{ABS}$ não significa que danos mais graves possam ter ocorrido na estrutura do complexo fotoquímico da membrana tilacóide. Segundo Force et al. (2003), a fotoinibição é mais precisamente identificada pelo declínio $\mathrm{em} \mathrm{ET}_{0} / \mathrm{TR}_{0}$ do que pelo declínio na razão $\mathrm{F}_{\mathrm{V}} / \mathrm{F}_{\mathrm{m}}\left(\mathrm{TR}_{0} / \mathrm{ABS}\right)$. Assim, plantas de $C$. guianensis com 14 dias de supressão da irrigação já poderiam estar sofrendo inibição, uma vez que as respostas de $\mathrm{ET}_{0} / \mathrm{TR}_{0}$, com a desidratação, foram menores do que as repostas de $\mathrm{TR}_{0} / \mathrm{ABS}$. Segundo Flexas \& Medrano (2002), a redução na taxa de $\mathrm{g}_{\mathrm{s}}$, com o aumento do deficit hídrico, implica redução da atividade fotoquímica, assim, a fotoinibição eventualmente ocorre sob condições de seca mais severa e quase completo fechamento estomático, fato ocorrido neste trabalho. Além disso, 56\% de redução no $\mathrm{PI}_{\mathrm{ABS}}$ também contribuem para essa afirmativa, uma vez que essa variável vem sendo considerada como um parâmetro mais sensível para a detecção e quantificação de estresse em plantas, do que a resposta da eficiência máxima do fotossistema II (Christen et al., 2007; Oukarroum et al., 2007).

Quando os valores de A se tornaram extremamente baixos, ou seja, próximos a zero, determinou-se o $\Psi_{\mathrm{w}}$ nas plantas jovens de $C$. guianensis. Os valores foram $-0,7$ e $-3,4 \mathrm{MPa}$ nas plantas irrigadas e não irrigadas, respectivamente. Após oito dias de reidratação das plantas não irrigadas, o valor do $\Psi_{\mathrm{w}}$ foi de $-0,5 \mathrm{MPa}$, semelhante aos valores observados nas plantas irrigadas. Isso demonstra que as plantas sob estresse hídrico não sofreram danos irreversíveis ou mesmo alterações mais profundas com a desidratação, indicando que essa espécie pode superar deficiência hídrica até valores próximos de $-4 \mathrm{MPa}$. Em plantas jovens de M. guianensis, a recuperação do potencial hídrico foliar ocorreu após quatro dias de reidratação (Liberato et al., 2006). No presente trabalho, não foi possível afirmar quando as plantas de $C$. guianensis já poderiam estar sob condições hídricas adequadas, uma vez que não foi possível fazer o acompanhamento do potencial hídrico, pelo fato de as plantas apresentarem poucas folhas em razão da deficiência hídrica induzida.

Considerando que as plantas não irrigadas apresentaram redução nas respostas das trocas gasosas 14 dias após a supressão da irrigação, verificou-se uma rápida recuperação das plantas de $C$. guianensis, no período de quatro dias de reidratação, com exceção da taxa de A, em que a recuperação foi mais evidente com oito dias de reidratação (Figura 3). Contudo, em quatro dias de irrigação, o valor de A $\left(2,3 \mu \mathrm{mol} \mathrm{m} \mathrm{m}^{-2} \mathrm{~s}^{-1}\right)$ já apresentava tendência de recuperação $(46 \%)$, porém, não significativa, comparado com o tratamento com irrigação $\left(4,8 \mu \mathrm{mol} \mathrm{m} \mathrm{m}^{-2} \mathrm{~s}^{-1}\right)$. Em relação às respostas de $g_{s}$, quatro dias de reidratação foram suficientes para os tratamentos apresentarem semelhanças estatísticas, com uma recuperação de $43 \%$, comparado com a leitura final $\left(6,4 \mathrm{mmol} \mathrm{m}^{-2} \mathrm{~s}^{-1}\right)$. Por sua vez, os valores de E, também, exibiram recuperação no quarto dia de reidratação, porém, de $68 \%$, comparado com a leitura final $\left(1,1 \mathrm{mmol} \mathrm{m}^{-2} \mathrm{~s}^{-1}\right)$.

Em plantas de $M$. guianensis, a recuperação da taxa de A se deu em seis dias (Liberato et al., 2006), diferente das respostas de C. guianensis neste experimento, onde a recuperação se deu em oito dias. Contudo, para as taxas de $g_{s}$ e $E$ a recuperação foi no quarto dia, no presente trabalho, semelhantemente à recuperação de M. guianensis em experimento de supressão de água. Esse comportamento, em que a resposta da taxa de $\mathrm{g}_{\mathrm{s}}$ ocorre de maneira mais rápida do que a resposta da
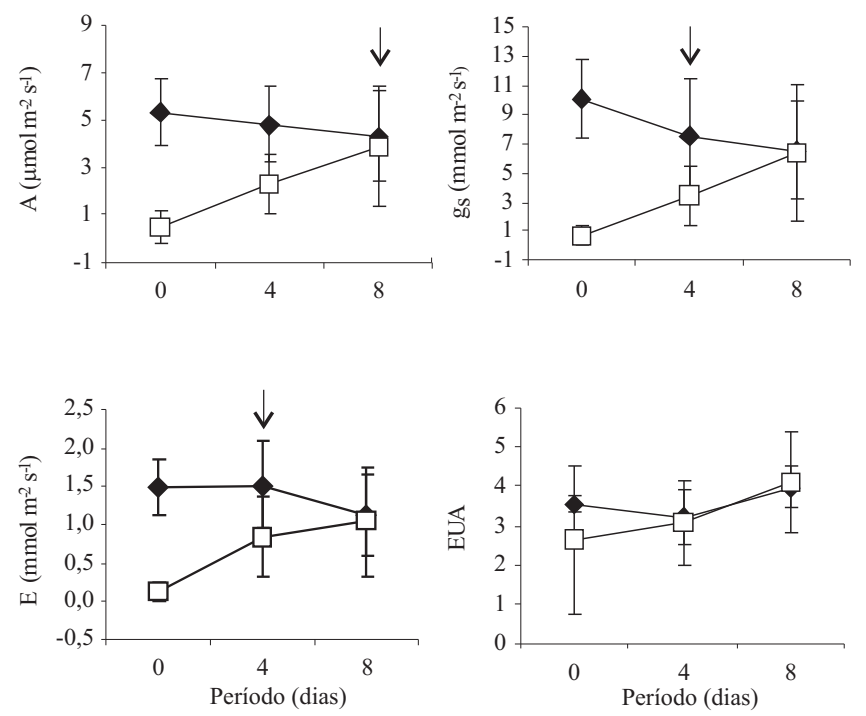

Figura 3. Fotossíntese líquida (A), condutância estomática (gs), transpiração (E) e eficiência no uso da água (EUA) de plantas jovens de Carapa guianensis irrigadas $(\diamond)$ e não irrigadas submetidas a reidratação $(\square)$. Os valores são médias e as linhas verticais indicam os desvios-padrão. A seta indica que os tratamentos já não apresentavam diferença significativa pelo teste $t$, a $5 \%$ de probabilidade $(n=5)$. 
taxa de A, confirma o fato de os estômatos atuarem como eficiente mecanismo de entrada de $\mathrm{CO}_{2}$, para manter elevada a concentração de $\mathrm{CO}_{2}$ intercelular utilizado na fase bioquímica da fotossíntese, o que justifica a demora na resposta da taxa de A neste tipo de experimento de reidratação de plantas (Lawlor, 2002). Quanto aos valores da eficiência no uso da água (EUA), não se observou diferença significativa entre os tratamentos ao longo do período do experimento de reidratação da plantas jovens de $C$. guianensis (Figura 3).

Nas plantas jovens de C. guianensis, foi observada recuperação de $69 \%$ apenas para $\mathrm{ET}_{0} / \mathrm{TR}_{0}$, após quatro dias de reidratação, comparado com o tratamento irrigado. Contudo, para as variáveis $\mathrm{TR}_{0} / \mathrm{ABS}, \mathrm{ET}_{0} / \mathrm{ABS}$ e $\mathrm{PI}_{\mathrm{ABS}}$, somente foram observadas recuperações significativas (73, 68 e 52\%, respectivamente) após oito dias de reidratação, comparado com o tratamento irrigado (Figura 4).
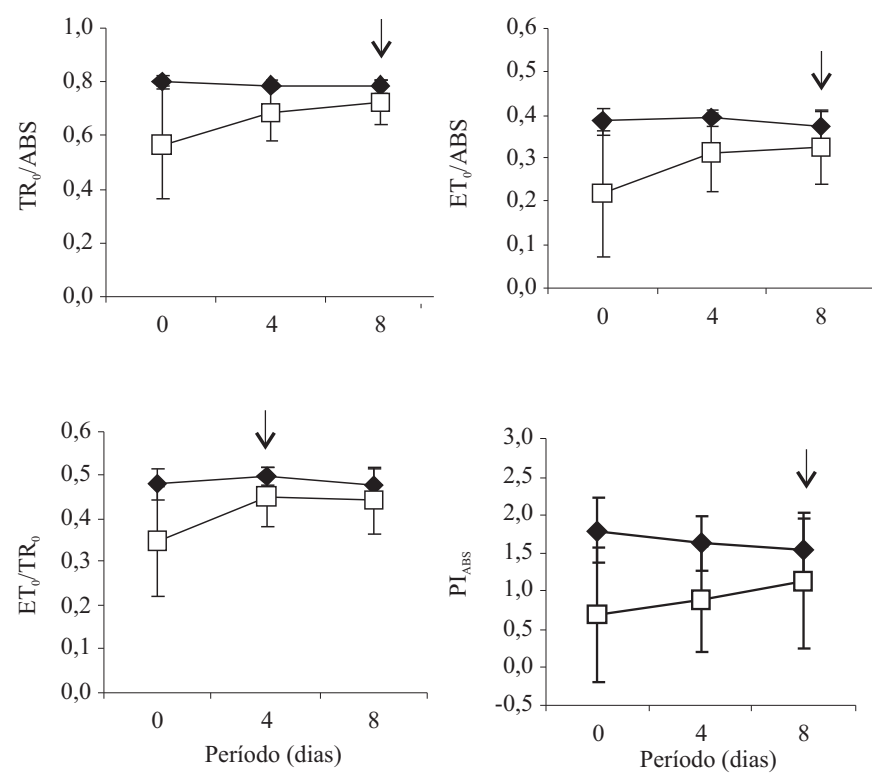

Figura 4. Eficiência máxima do fotossistema II ( $\left.\mathrm{TR}_{0} / \mathrm{ABS}\right)$, probabilidade de um fóton absorvido mover um elétron após a quinona $\mathrm{A}\left(\mathrm{ET}_{0} / \mathrm{ABS}\right)$, probabilidade de um elétron capturado ser transportado após a quinona $\mathrm{A}\left(\mathrm{ET}_{0} / \mathrm{TR}_{0}\right)$ e o índice de desempenho $\left(\mathrm{PI}_{\mathrm{ABS}}\right)$ de plantas jovens de Carapa guianensis irrigadas ( ) e não irrigadas submetidas a reidratação ( $\square$ ). Os valores são médias e as linhas verticais indicam os desvios-padrão. A seta indica que os tratamentos já não apresentavam diferença significativa pelo teste t, a 5\% de probabilidade $(\mathrm{n}=5)$.
Mesmo tendo sido observada boa recuperação da variável $\mathrm{TR}_{0} / \mathrm{ABS}$, após a reidratação das plantas não irrigadas, verificou-se que essa variável não apresentou informações conclusivas sobre a tolerância ao estresse hídrico, à semelhança do que foi observado em plantas de Hordeum vulgare L. submetidas à deficiência hídrica e à reidratação (Oukarroum et al., 2007). Contudo, a recuperação observada pela variável $\mathrm{PI}_{\mathrm{ABS}}$, após a reidratação das plantas não irrigadas, indica que elas não sofreram danos irreversíveis no aparato fotossintético, uma vez que essa variável tem sido considerada mais eficiente na verificação de estresse em plantas do que a razão $\mathrm{F}_{\mathrm{v}} / \mathrm{F}_{\mathrm{m}}$. Já a recuperação de $\mathrm{ET}_{0} / \mathrm{TR}_{0}$ no quarto dia de reidratação pode significar que processos como a fotorrespiração estejam ocorrendo, fato que contribui para o restabelecimento do fluxo de elétrons, mesmo antes do aumento da atividade fotossintética em razão da restrição do fluxo de $\mathrm{CO}_{2}$ (Osmond et al., 1997).

\section{Conclusões}

1. Os indicadores de fotossíntese de plantas jovens de Carapa guianensis decrescem fortemente sob deficit hídrico aos 21 dias; a recuperação desses indicadores ocorre no período de até oito dias, restabelecendo todas as características fotossintéticas das plantas irrigadas.

2. Plantas jovens de $C$. guianensis são tolerantes ao estresse hídrico durante o período de 21 dias, e atribuise esse comportamento, principalmente, à eficiente regulação estomática.

3. A reidratação permite rápida recuperação das plantas jovens de C. guianensis, o que demonstra boa plasticidade fisiológica dessa espécie, quando submetida ao estresse hídrico.

\section{Agradecimentos}

Ao Conselho Nacional de Desenvolvimento Científico e Tecnológico, pela concessão das bolsas.

\section{Referências}

CALBO, M.E.R.; MORAES, J.A.P.V. Efeitos da deficiência de água em plantas de Euterpe oleracea (açaí). Revista Brasileira de Botânica, v.23, p.225-230, 2000.

CASPER, C.; EICKMEIER, W.G.; OSMOND, C.B. Changes of fluorescence and xanthophylls pigments during dehydration in the resurrection plant Selaginella lepidophylla in low and medium light intensities. Oecologia, v.94, p.528-533, 1993. 
CHAVES, M.M.; PEREIRA, J.S.; MAROCO, J.; RODRIGUES, M.L.; RICARDO, P.P.; OSÓRIO, M.L.; CARVALHO, I.; FARIA, T.; PINHEIRO, C. How plants cope with water stress in the field. Photosynthesis and growth. Annals of Botany, v.89, p.907-916, 2002.

CHRISTEN, D.; SCHÖNMANN, S.; JERMINI, M.; STRASSER, R.J.; DÉFAGO, G. Characterization and early detection of grapevine (Vitis vinifera) stress responses to esca disease by in situ chlorophyll fluorescence and comparison with drought stress. Environmental and Experimental Botany, v.60, p.504-514, 2007.

COSTA, G.F.; MARENCO, R.A. Fotossíntese, condutância estomática e potencial hídrico foliar em árvores jovens de andiroba (Carapa guianensis). Acta Amazonica, v.37, p.229-234, 2007.

FARQUHAR, G.D.; SHARKEY, T.D. Stomatal conductance and photosynthesis. Annual Review of Plant Physiology, v.33, p.317345, 1982.

FISCH, G.; MARENGO, J.A.; NOBRE, C.A. Uma revisão geral sobre o clima da Amazônia. Acta Amazonica, v.28, p.101-126, 1998.

FLEXAS, J.; MEDRANO, H. Drought-inhibition of photosynthesis in $\mathrm{C}_{3}$ plants: stomatal and non-stomatal limitations revisited. Annals of Botany, v.89, p.183-189, 2002.

FORCE, L.; CRITCHLEY, C.; RENSEN, J.J.S. New fluorescence parameters for monitoring photosynthesis in plants. Photosynthesis Research, v.78, p.17-33, 2003.

GEBREKIRSTOS,A.;TEKETAY,D.;FETENE,M.;MITLOHNER, R. Adaptation of five co-occurring tree and shrub species to water stress and its implication in restoration of degraded lands. Forest Ecology and Management, v.229, p.259-267, 2006.

GONÇALVES, J.F.C.; BARRETO, D.C.S.; SANTOS JUNIOR, U.M.; FERNANDES, A.V.; SAMPAIO, P.T.B.; BUCKERIDGE, M.S. Growth, photosynthesis and stress indicators in young rosewood plants (Aniba rosaeodora Ducke) under different light intensities. Brazilian Journal of Plant Physiology, v.17, p.325334, 2005.

LAWLOR, D.W. Limitation to photosynthesis in water-stressed leaves: stomata vs. metabolism and the role of ATP. Annals of Botany, v.89, p.871-885, 2002.

LECHNER, L.; PEREYRA-IRUJO, G.A.; GRANIER, C.; AGUIRREZÁBAL, L.A.N. Rewatering plants after a long waterdeficit treatment reveals that leaf epidermal cells retain their ability to expand after the leaf has apparently reached its final size. Annals of Botany, v.101, p.1007-1015, 2008.

LIBERATO, M.A.R.; GONÇALVES, J.F.C.; CHEVREUIL, L.R.; NINA JUNIOR, A.R.; FERNANDES, A.V.; SANTOS JUNIOR, U.M. Leaf water potential, gas exchange and chlorophyll $a$ fluorescence in acariquara seedlings (Minquartia guianensis Aubl.) under water stress and recovery. Brazilian Journal of Plant Physiology, v.18, p.315-323, 2006.

MENDONCA, A.P.; FERRAZ, I.D.K. Óleo de andiroba: o processo tradicional da extração, uso e aspectos sociais no Estado do Amazonas, Brasil. Acta Amazonica, v.37, p.353-364, 2007.

O'LEARY, J.W. A critical evaluation of tissue-immersion methods for measurement of plant water potencial. Ohio Journal of Science, v.70, p.34-38, 1970.

OLIVEIRA, M.A.J.; BOVI, M.L.A.; MACHADO, E.C.; GOMES, M.M.A.; HABERMANN, G.; RODRIGUES, J.D. Fotossíntese, condutância estomática e transpiração em pupunheira sob deficiência hídrica. Scientia Agricola, v.59, p.59-63, 2002.

OSMOND, B.; BADGER, M.; MAXWELL, K.; BJÖRKMAN, O.; LEEGOOD, R. Too many photons: photorespiration, photoinhibition and photooxidation. Trends in Plant Science, v.2, p.119-120, 1997.

OUKARROUM, A.; EL MADIDI, S.; SCHANSKER, G.; STRASSER, R.J. Probing the responses of barley cultivars (Hordeum vulgare L.) by chlorophyll a fluorescence OLKJIP under drought stress and re-watering. Environmental and Experimental Botany, v.60, p.438-446, 2007.

REINHARDT, G.; RETTENMAIER, N.; GÃRTNER, S.; PASTOWSKI, A. Rain forest for biodiesel? Ecological effects of using palm oil as a sourse of energy. Frankfurt: WWF Germany, 2007. 50p.

SANTOS JUNIOR, U.M.; GONÇALVES, J.F.C.; FELDPAUSCH, T.R. Growth, leaf nutrient concentration and photosynthetic nutrient use efficiency in tropical tree species planted in degraded areas in central Amazonia. Forest Ecology and Management, v.226, p.299-309, 2006.

SAS INSTITUTE. JMP Version 4.0. Cary: SAS Institute, 2000.

SILVA, E.C.; NOGUEIRA, R.J.M.C.; AZEVEDO NETO, A.D.; SANTOS, V.F. Comportamento estomático e potencial da água da folha em três espécies lenhosas cultivadas sob estresse hídrico. Acta Botanica Brasilica, v.17, p.231-246, 2003.

SOUZA, C.R.; SOARES, A.M.; REGINA, M.A. Trocas gasosas de mudas de videira, obtidas por dois porta-enxertos, submetidas à deficiência hídrica. Pesquisa Agropecuária Brasileira, v.36, p.1221-1230, 2001.

SRIVASTAVA, A.; STRASSER, R.J.; GOVINDJEE. Greening of peas: parallel measurements of $77 \mathrm{~K}$ emission spectra, OJIP chlorophyll $a$ fluorescence transient, period four oscillation of the initial fluorescence level, delayed light emission, and P700. Photosynthetica, v.37, p.365-392, 1999.

TURNER, N.C. Techniques and experimental approaches for the measurement of plant water status. Plant and Soil, v.58, p.339-366, 1981.

$\overline{\text { Recebido em } 23 \text { de abril de } 2008 \text { e aprovado em } 22 \text { de dezembro de } 2008}$ 